\title{
Les Simulies (Diptera Simuliidae) du Sud-Ouest de l'Europe : le crénal et l'épirhithral
}

\author{
G. Vinçon 1 \\ M. Clergue-Gazeau ${ }^{2}$
}

Mots-clés : Simuliidae, crénal, épirhithral, Sud-Ouest de l'Europe.

De 1984 à 1991, 114 stations du crénal et de l'épirhithral ont été régulièrement prospectées dans les hauts massifs montagneux du Sud-Ouest de l'Europe (Alpes françaises et italiennes, Pyrénées et principales sierras de la Péninsule Ibérique).

Parmi les 33 espèces recensées, peu sont crénophiles : $S$. (N.) carpathicum, $P$. (P.) latimucro et $S$. ( $N$.) oligotuberculatum se développent en très haute altitude; $S$. (N.) vernum, $S$. $(N$.) brevidens, $S$. (N.) quasidecolletum et $S$. (E.) petricolum en haute montagne; $S$. (N.) costatum, $S$. (R.) lamachi et $P$. (P.) tomosvaryi en moyenne montagne et piémont ; la majorité d'entre elles fréquentent également l'épirhithral. Des espèces peu alticoles dans les Alpes ou les Pyrénées peuvent atteindre des altitudes de plus en plus élevées vers le sud de l'Europe (sierras andalouses en Espagne, Haut-Atlas marocain), par exemple : $S$. (N.) costatum, $S$. (S.) intermedium, $S$. (R.) lamachi.

\section{Simuliidae (Diptera) from south west Europe : the crenal and the epirhithral}

Keywords : Simuliidae, crenal, epirhithral, South West Europe.

Between 1984 and 1991, 114 stations belonging to the crenal or the epirhithral zone have been regularly visited in the high South West European mountains (French and Italian Alps, Pyrenees and Iberic Sierras).

Biogeographical and ecological observations.

Among the 33 species collected, the most typical of the crenal zone are : $P$. (P.) latimucro, $S$. (N.) carpathicum, and $S$. (N.) oligotuberculatum that live at very high altitude; $S$. (N.) vernum, $S$. (N.) brevidens, $S$. (N.) quasidecolletum and $S$. (E.) petricolum in high mountain ous regions; $S$. (N.) costatum, $S$. (R.) lamachi and $P$. (P.) tomosvaryi at lower elevations and in the zone of the foothills. Species that live at low elevations in the Alps and Pyrenees can develop at higher altitudes in Andalusia (Spain) or High Atlas (Morocco), for exemple : $S$. (N.) costatum, $S$. (S.) intermedium, $S$. (R.) lamachi.

\section{Introduction}

Des études hydrobiologiques récentes (ClergueGazeau \& Gazagnes 1986, Clergue-Gazeau 1987, Vinçon \& Clergue-Gazeau 1988), ont mis en évidence la faible diversification du peuplement simuliidien dans les sources et leurs émissaires. Afin de compléter ces observations, des récoltes ont été effectuées dans les principaux massifs montagneux

1. 38, bis rue du Drac, 38000 Grenoble, France.

2. Laboratoire d'Hydrobiologie, URA 695 CNRS, Université Paul Sabatier, 118 route de Narbonne, 31062 Toulouse Cedex, France. du Sud-Ouest de l'Europe : Alpes françaises et italiennes, Pyrénées, sierras de la Péninsule Ibérique. Les biotopes montagnards de haute altitude ont été spécialement prospectés ; difficiles d'accès, ils sont encore insuffisamment connus.

Ces recherches complètent le travail de Dorier (1962-63) sur les simulies des Alpes françaises, notamment pour les biocénoses du crénal d'altitude.

Il n'existe pas, par contre, de travail de synthèse sur la faune simulidienne des Pyrénées. Récemment, d'importants travaux ont été réalisés sur l'écologie de ces Diptères dans différents réseaux hydrographiques de cette chaîne (Lavandier 1976, GonzálezPeña 1983, Clergue-Gazeau \& Gazagnes 1986, Vinçon et Clergue-Gazeau 1988). 
En ce qui concerne la Péninsule Ibérique, des publications nous renseignent sur la faune simuliidienne d'un cours d'eau de la Sierra Morena (González-Peña \& al. 1986) ou de plusieurs réseaux hydrographiques du Portugal, et de diverses régions de l'Espagne (Beaucournu-Saguez 1972, 1975a, 1975b, Santos-Gracio 1981, González-Peña 1990). Notre attention s'est portée sur les principaux ensembles montagneux ibériques mais également sur des sierras isolées encore peu connues.

\section{Les stations étudiées}

La liste des 114 stations prospectées dans les Alpes occidentales, les Pyrénées et la Péninsule Ibérique (Fig. 1) est récapitulée sur les tableaux 1, 2 et 3.

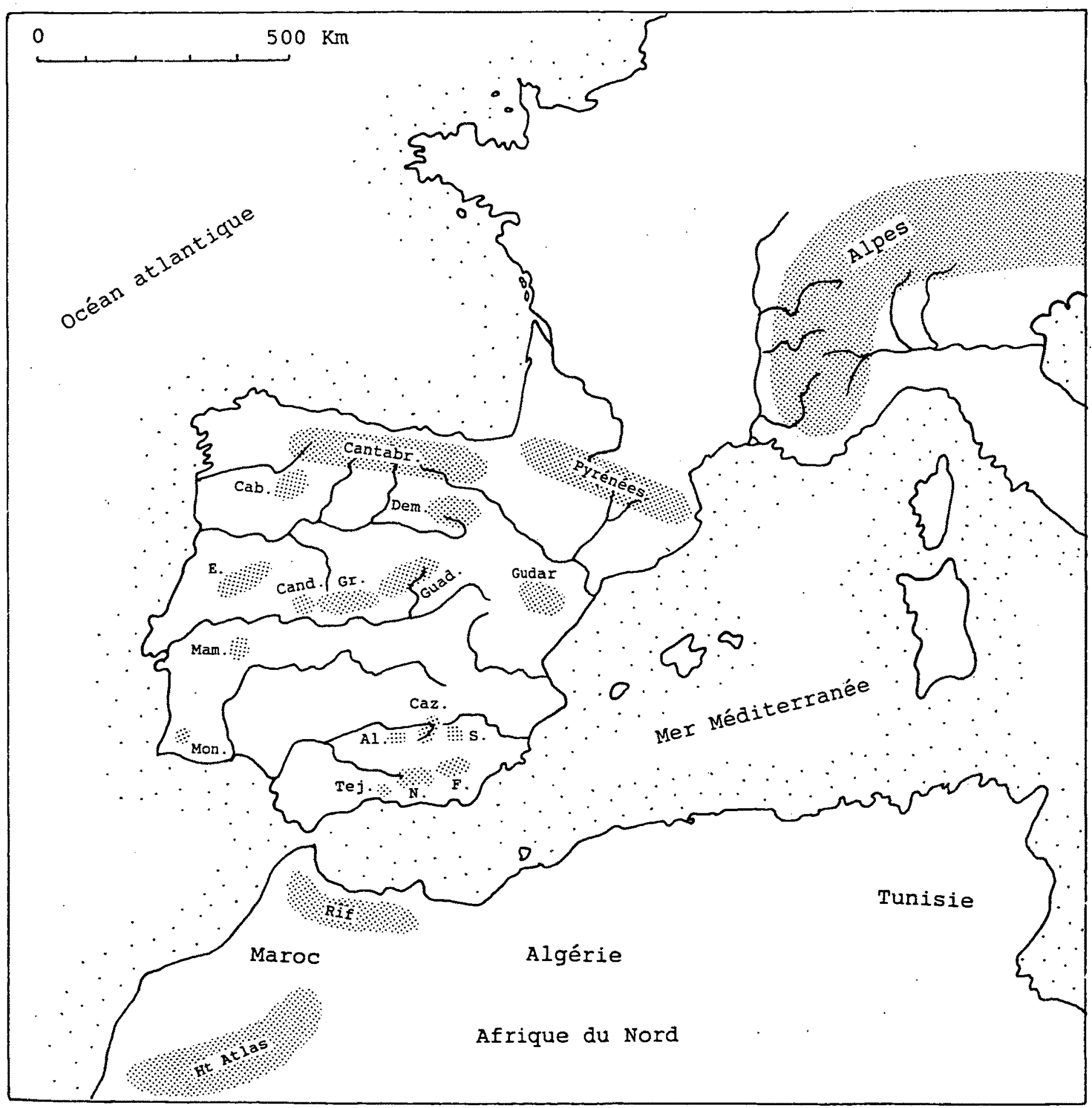

Fig. 1. Les massifs montagneux prospectés : Alpes françaises et italiennes, Pyrénées et Sierras ibériques.

Fig. 1. The mountainous regions examined : French and Italian Alps, Pyrenees and Iberic Sierras. 


\subsection{Alpes occidentales (Tableau 1)}

42 stations (st.) ont été prospectées : 37 en France (massifs de la Chartreuse, de l'Oisans, du Vercors, du Queyras et du Mercantour) et 5 en Italie dans le Piémont ( 1 dans la haute vallée de la Sésia et 4 dans la vallée de la Stura di Demonte).

$45 \%$ de ces stations appartiennent au crénal ; $33 \%$ sont situées au-dessus de $2000 \mathrm{~m}$ et la plus élevée atteint $2700 \mathrm{~m}$, dans le Queyras.

\subsection{Pyrénées (Tableau 2)}

Les 39 stations sont réparties tout au long de la chaîne et sur les deux versants.

$56 \%$ des stations appartiennent à la zone du crénal ; $62 \%$ sont situées au-dessus de $2000 \mathrm{~m}$ et la plus élevée se trouve en Andorre, à $2700 \mathrm{~m}$. 5 stations de la vallée d'Ossau (Pyrénées-Atlantiques) et 2 de la haute vallée du Rio Gallego (Aragon), bien qu'ayant fait l'objet d'un travail précédent (Vinçon \& Clergue-Gazeau 1988), ont été incluses dans cette étude car elles précisent la répartition des espèces crénophiles dans la partie occidentale des Pyrénées.

\subsection{Péninsule Ibérique (Tableau 3)}

32 stations ont été prospectées de 1987 à 1991, dans 14 ensembles montagneux. Parmi elles, 19 appartiennent à la zone du crénal $(59 \%)$; la plus élevée atteint $3000 \mathrm{~m}$, dans la Sierra Nevada.

Les sierras visitées, du nord vers le sud, appartiennent à quatre principaux systèmes montagneux (Fig. 1) :

- Cordillère cantabrique et Montes de Leon (Sierra de la Cabrera),

- Cordillère ibérique (Sierras : Demanda, Gudar et Javalambre),

- Cordillère transversale, sensu Aubert 1963 (Sierras : Guadarrama et Candelario),

- Cordillère bétique (Sierras : Sagra, Cazorla, Almaden, Filabres, Nevada et Tejeda).

Quelques serras Portugaises isolées et moins connues ont également été prospectées :

- Serra de Mamede, centre du Portugal, point culminant $1028 \mathrm{~m}$,

- Serra de Monchique, extrémité sud-ouest du Portugal, point culminant $901 \mathrm{~m}$.

\section{Méthodes et périodes de récoltes}

Les nymphes et les larves ont été récoltées à vue dans les différents biotopes de la station (rochers, végétation immergée) ; elles sont conservées dans de l'alcool à $70^{\circ}$. La durée moyenne d'un prélèvement benthique est de 15 minutes.

Les adultes ont été capturés au filet ou extraits de nymphes âgées. Le nombre de prélèvements par station et leur période sont indiqués dans les tableaux 1,2 et 3 .

Les stations alpines et pyrénéennes ont été prospectées pour la plupart à 3 saisons (printemps, été et automne). En Espagne, nous avons effectué deux séries de récoltes printanières (nord de l'Espagne et Andalousie) et plusieurs estivales dans différentes régions de la péninsule, mais beaucoup de stations n'ont été visitées qu'une seule fois.

\section{La faune des Simuliidae}

\subsection{Généralités}

La liste faunistique globale (Tableau 4) comprend 33 espèces réparties en 2 genres et 8 sous-genres, selon la classification de Crosskey (1988). 25 ont été recensées dans les Alpes, 25 dans les Pyrénées et 19 dans la Péninsule Ibérique (Pyrénées exclues).

Dans les listes faunistiques détaillées (Tableaux 5 , 6 et 7), les stations sont indiquées par leur localisation (département, province ou pays, nom du cours d'eau, altitude). Les espèces, nommées par leur code (cf. Tableau 4), sont réparties en 5 classes d'abondance. La diversité spécifique (d. sp.) souligne la richesse de certaines stations mais dépend aussi du nombre de prélèvements effectués ; ainsi les stations de la Péninsule Ibérique, moins souvent visitées que celles des Alpes ou des Pyrénées, ont une diversité nettement sous-estimée. Les fréquences dans le crénal et dans l'épirhithral permettent, par comparaison, d'estimer le caractère crénophile des espèces.

\subsection{Espèces présentant un intérêt biogéographique \\ a) Espèces alpines}

Parmi les espèces recensées dans les Alpes occidentales, on remarque plus particulièrement la présence de deux taxons encore inconnus de France : $S$. (N.) oligotuberculatum et $S$. (T.) graium. 
Tableau 1. Caractéristiques des stations prospectées dans les Alpes occidentales (françaises et italiennes), du nord au sud.

Table 1. Characteristics of the sampling stations in the occidental Alps, from north to south.

\begin{tabular}{|c|c|c|c|c|c|c|c|c|c|c|}
\hline LOC & $N^{\circ}$ & COURS D'EAU & CRE & LAPI & ALT & VIC & LARG & VEGETATION BORDANTE & MOIS & $\mathbb{N}$ \\
\hline SAVOIE & 73 & af. Reclus (< col Petit St Bernard) & * & $45^{\circ} 40$ & 2100 & $\mathrm{R}$ & $1-2$ & pel. alpine & 05 à 10 & 5 \\
\hline SAVOIE & 73 & af. Isère (à Ste Foy Tarentaise) & & $45^{\circ} 40$ & 1000 & $\mathrm{R}$ & $2-3$ & feuillus & 05 à 10 & 5 \\
\hline SAVOIB & 73 & t. des Moulins (af. Isère) & & $45^{\circ} 40^{\circ}$ & 1400 & $\mathrm{R}$ & $3-5$ & feuillus & 05 à 10 & 5 \\
\hline SAVOIE & 73 & r. de la Lenta (< col de l'Iseran) & $\star$ & $45^{\circ} 20$ & 2300 & $\mathbb{R}$ & $1-2$ & pel. alpine & 05 à 10 & 5 \\
\hline ISERE & 38 & Vence au Sappey (Chartreuse) & & $45^{\circ} 20$ & 1000 & M & $2-4$ & feuillus & & 6 \\
\hline ISERR & 38 & Vence (Chartreuse) & & $45^{\circ} 20$ & 600 & $\mathbb{R}$ & $2-4$ & & & 4 \\
\hline ISERR & 38 & Vence (Chartreuse) & & $45^{\circ} 20$ & 240 & $\mathbf{R}$ & $5-7$ & pel. + feuillus & 04 à 09 & 3 \\
\hline ISERR & 38 & af. Romanche (La Grange) & & $45^{\circ}$ & 1000 & $\mathrm{R}$ & $1-2$ & feuillus & 04 à 10 & 6 \\
\hline ISERE & 38 & Rif Garcin (Taillefer) & $\star$ & $45^{\circ}$ & 2030 & $M$ & $2-4$ & pel. alpine & 6 à 10 & 2 \\
\hline ISERE & 38 & af. Plumet (af. Romanche) & & $45^{\circ} 10$ & 1900 & $\mathbb{R}$ & $1-2$ & pel. subalpine & 15 à 10 & 2 \\
\hline ISERE & 38 & Drevenne, af. Isère (Vercors) & * & $45^{\circ} 10$ & 1000 & $y$ & $1-2$ & hêtraie montagnarde & 04 à 09 & 3 \\
\hline ISRRE & 38 & Drevenne, af. Isère (Vercors) & & $45^{\circ} 10$ & 700 & $\mathrm{R}$ & $2-3$ & hêtraie montagnarde & 04 à 09 & 3 \\
\hline ISERR & 38 & Voroize, af. Isère (Vercors) & $\star$ & $45^{\circ} 10$ & 600 & M & $1-2$ & feuillus & 03 à 05 & 2 \\
\hline ISERR & 38 & Furon (Vercors) & * & $45^{\circ} 10$ & 1300 & $\mathrm{R}$ & $1-2$ & feuillus & 03 à 09 & 4 \\
\hline IERR & 38 & $r$. des Pellas $<$ Mont $A$ & * & $44^{\circ} 50$ & 1400 & $\mathrm{R}$ & $1-2$ & hêtraie mon & 03 à 09 & 5 \\
\hline RRP & 38 & r. des Pellas $<$ Mon & & $44^{\circ} 50$ & 1200 & 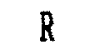 & $2-4$ & thraie mon & 03 à 09 & 4 \\
\hline ERR & 38 & r. af, $\mathrm{E}$ & & $44^{\circ} 40$ & 1100 & H & $1-2$ & fevillus & 4 à 09 & 4 \\
\hline ISERR & 38 & Jocou, af. Ebron (col Croix Haute) & * & $44^{\circ} 40$ & 1500 & 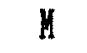 & $1-3$ & hêtraie montagnarde & 04 à 09 & 6 \\
\hline DROME & 26 & Archiane (sud du Vercors) & & $44^{\circ} 40$ & 1300 & $\mathbf{R}$ & $2-3$ & aie mon & 05 à 09 & 5 \\
\hline COKR & 26 & Archiane & & $44^{\circ} 40$ & 600 & $\mathrm{R}$ & $4-6$ & illius & 04 à 09 & 5 \\
\hline U-ALP & 05 & Bléronnet /c & & 50 & 1800 & H & $2-4$ & subalp. & 06 à 07 & 2 \\
\hline U-ALP & 05 & t. de $B C$ & $\star$ & 50 & 2300 & $\mathbb{R}$ & $1-2$ & oustes & 6 à 07 & 4 \\
\hline U-ALP & 05 & af. Bou & * & 40 & 2700 & L & $1-2$ & he & 09 & 1 \\
\hline U-ALP & 05 & t. de & * & $44^{\circ} 40^{\circ}-2-2$ & 2400 & $\mathbb{R}$ & $2-4$ & pel. alp. & 05 à 11 & 6 \\
\hline U-ALP & 05 & Aigue & * & $44^{\circ} 40$ & 2600 & $M$ & $1-2$ & pel. alpine & 09 & 1 \\
\hline HAU-ALP & 05 & af. $\mathrm{Cl}$ & * & $44^{\circ} 30$ & 2100 & $R$ & $1-2$ & el. alp. & 05 à 09 & 5 \\
\hline$L-H-P R$ & 04 & af. Mounal & & $44^{\circ} 30$ & 2000 & $\mathbb{R}$ & $2-4$ & pel. alpir & 05 à 10 & 5 \\
\hline $\mathrm{L}-\mathrm{H}-\mathrm{PR}$ & 04 & Oronaye & & $44^{\circ} 20$ & 2000 & $\mathbb{R}$ & $3-5$ & pel. alpine + & 05 à 10 & 4 \\
\hline $\mathrm{AL}-\mathrm{H}-\mathrm{PR}$ & 04 & af. Bachelar & * & $44^{\circ} 20^{\circ}-1-10$ & 2200 & $\mathrm{R}$ & $1-2$ & pel. alpine & 06 à 09 & 2 \\
\hline ALL-H-PR & 04 & af. Bache & & $44^{\circ} 20$ & 1800 & $\mathbf{R}$ & $3-5$ & pel. subalp. & 06 à 09 & 2 \\
\hline ALP-MAR & 06 & Tínée (co) & * & $44^{\circ} 20$ & 2300 & II & $1-2$ & pel. alpine + résineux & 08 à 09 & 2 \\
\hline ALP-MAR & 06 & de la Moutière) & & $44^{\circ} 20$ & 2000 & $\mathbb{R}$ & $3-5$ & forêt de résineux & 08 à 09 & 2 \\
\hline ALP_WAR & 06 & af. Vésubie (> la Bollène) & * & $44^{\circ}$ & 1500 & $\mathbf{R}$ & $1-2$ & feuillus + résineux & 05 à 09 & 3 \\
\hline ALP-MAR & 06 & af. Bévéra (af. Roya) & * & $44^{\circ}$ & 1700 & $\mathbb{H}$ & $1-2$ & pel. subalpine & 06 & 1 \\
\hline ALP-MAR & 06 & Refrei, af. Roya (col Tende) & * & $44^{\circ} 10$ & 1900 & II & $2-3$ & pel. subalp. + fevillus & 05 à 09 & 3 \\
\hline ALP-MAR & 06 & Caramagne (af. Roya) & & $44^{\circ} 10$ & 1400 & $\mathbb{R}$ & $1-2$ & feuillus & 05 à 09 & \\
\hline ALP-MAR & 06 & Roya (au-dessus de Tende) & & $44^{\circ} 10$ & 850 & $\mathrm{R}$ & $4-6$ & feuillus & 04 & 1 \\
\hline ITALIE & & af. Sermenza à Rima (af. Sesia) & & $45^{\circ} 50$ & $1400^{\circ}$ & $\mathbf{R}$ & $1-2$ & hêtraie montagnarde & 06 & 1 \\
\hline ITALIE & & af. Stura di Demonte & & $44^{\circ} 20$ & 1900 & $\mathrm{R}$ & $2-3$ & & 06 & 1 \\
\hline ITALIE & & Prati della Chiesa (col Tende) & & $44^{\circ} 10$ & 1900 & $\mathbb{R}$ & $2-4$ & & 10 & 1 \\
\hline ITALIR & & af. Prati della chiesa (col Tende) & * & $44^{\circ} 10$ & 2000 & $\mathrm{R}$ & $1-2$ & buissons de feuillus & 06 à 09 & 2 \\
\hline ITALIE & & af. Prati della Chiesa (col Tende) & & $44^{\circ} 10$ & 1600 & $\mathbb{R}$ & $2-4$ & feuillus & 05 à 10 & 4 \\
\hline
\end{tabular}

LOC : Localité, N du département, CRE : crenon, LATI : latitude, ALTI : Altitude, WMC : vitesse moyenne du courant, LARG : largeur du lit, $N$ : nombre de prélèvenents, af. : affluent, dév. : déversoir, pel. : pelouse

$\mathbb{R}$ : très rapide, $R$ : rapide, $M:$ modéré, $L$ : lent

HAU-ALP : Hautes-Älpes, AL-H-PR : Alpes de Haute Provence, ALP-MAR : Alpes-Maritimes 
Tableau 2. Caractéristiques des stations prospectées dans la chaîne pyrénéenne, sur les versants français et espagnol (Catalogne, Aragon et Navarre) et en Andorre.

Table 2. Characteristics of the sampling stations in the French and Spanish Pyrenees (Catalogne, Aragon, Navarra) and in Andorra.

\begin{tabular}{|c|c|c|c|c|c|c|c|c|c|c|}
\hline LOC & $\mathbb{N}^{\circ}$ & COURS D'EAU & CRE & LATI & ALTI & WIC & LARG & VEGETATTON BORDANTE & MOIS & N \\
\hline PYR-ORI & 66 & Llitéra (af. Têt) & * & $42^{\circ} 30$ & 2200 & $\mathrm{~L}$ & $2-4$ & pel. alpine & 07 à 09 & 2 \\
\hline PYR-ORI & 66 & Riberole (af. Têt) & * & $42^{\circ} 30$ & 2400 & $\mathbf{R}$ & $1-2$ & pel. alpine & 07 & 1 \\
\hline PYR-ORI & 66 & Eyne (af. Sègre) & * & $42^{\circ} 30$ & 2500 & $\mathbf{R}$ & $1-2$ & pel. alpine + arbustes & 07 & 1 \\
\hline PYR-ORI & 66 & Brr (af. Sègre) & * & $42^{\circ} 30$ & 2200 & $\mathbb{R}$ & $2-4$ & pel. alpine + arbustes & 07 à 09 & 3 \\
\hline PYR-ORI & 66 & Err (af. Sègre) & & $42^{\circ} 30$ & 1900 & $\mathrm{R}$ & $2-4$ & pel. subalpine & 05 à 09 & 4 \\
\hline AUDE & 11 & af. Aiguette (af. Aude) & * & $42^{\circ} 40$ & 2300 & L & $1-2$ & pel. alpine & 07 & 1 \\
\hline PYR-ORI & 66 & af. Grave, af. Têt (Carlit) & * & $42^{\circ} 40$ & 2425 & $\mathbf{R}$ & $1-2$ & pel. alpine & 07 & 1 \\
\hline PYR-ORI & 66 & Coume des Fourats (Carlit) & * & $42^{\circ} 40$ & 2400 & $\mathbb{T R}$ & $1-2$ & pel. alpine & 07 & 1 \\
\hline PYR-ORI & 66 & Mesclan d'Eygues (Carlit) & * & $42^{\circ} 40$ & 2350 & $n$ & $1-2$ & pel. alpine & 07 & 2 \\
\hline PYR-ORI & 66 & Courtal Rousseau (af. Sègre) & * & $42^{\circ} 40$ & 2400 & $\mathrm{~L}$ & $1-2$ & pel. alpine & 07 à 08 & 2 \\
\hline PYR-ORI & 66 & Courtal Rousseau (af. Sègre) & & $42^{\circ} 40$ & 2200 & $R$ & $2-4$ & pel. alpine & 07 à 08 & 2 \\
\hline PYR-ORI & 66 & En Garcia (af. Ariège) & * & $42^{\circ} 40$ & 2300 & $\mathbf{R}$ & $1-2$ & pel. alpine & 07 à 08 & 2. \\
\hline PYR=ORI & 66 & En Garcia (af. Ariège) & & $42^{\circ} 40$ & 2000 & $\mathrm{R}$ & $4-6$ & pel. subalpine & 07 à 09 & 3 \\
\hline CATALOG & & r. > Estana (sierra du Cadi) & * & $42^{\circ} 20$ & 1800 & $\mathbb{R}$ & $2-4$ & feuillus & 07 & 1 \\
\hline CATALOG & & r. > Estana (sierra du Cadi) & & $42^{\circ} 20$ & 1600 & $\mathrm{R}$ & $1-2$ & feuillus & 07 & 1 \\
\hline CATALOG & & Duran (af. Sègre) & * & $42^{\circ} 30$ & 2500 & $M$ & $1-2$ & pel. alpine & 07 & 1 \\
\hline CATALDG & & Duran (af. Sègre) & & $42^{\circ} 30$ & 2300 & $\mathbf{R}$ & $2-4$ & pel. alpine & 07 & 1 \\
\hline CAPALOG & & Puerto de Tosas (af. Sègre) & & $42^{\circ} 30$ & 1700 & $\mathbb{M}$ & $2-4$ & pel, montagn. feuillus & 08 à 09 & 2 \\
\hline ARIEGE & 09 & af. Ariège (avant Pas da la Case) & * & $42^{\circ} 30$ & 2200 & $\mathbb{R}$ & $1-3$ & pel, alpine & 07 à 08 & 3 \\
\hline ARIEGE & 09 & af. Ariège (avant Pas de la Case) & & $42^{\circ} 30$ & 1900 & $\mathrm{R}$ & $4-6$ & pel. subalpine & 07 à 09 & 4 \\
\hline ARIEGE & 09 & Sant Josep (af. Ariège) & & $42^{\circ} 30$ & 2000 & $\mathrm{R}$ & $1-2$ & pel. subalpine & 07 & 1 \\
\hline ANDORRB & & Tristaina (af. Valira d'ordino) & & $42^{\circ} 40$ & 2200 & $\mathrm{R}$ & $2-3$ & arbustes & 07 & 1 \\
\hline ANDORRE & & Coma Pedrosa (af. Valira del Nord) & * & $42^{\circ} 30$ & 2700 & $\mathrm{R}$ & $1-2$ & pel. alpine & 07 à 08 & 2 \\
\hline ANDORRR & & Coma Pedrosa (af. Valira del Nord) & & $42^{\circ} 30$ & 2550 & $\mathbb{R}$ & $2-4$ & pel. alpine & 07 à 08 & 2 \\
\hline ANDORRE & & Cona Pedrosa (af. Valira del Nord) & & $42^{\circ} 30$ & 2400 & $\mathrm{R}$ & $1-2$ & pel, alpine & 07 à 08 & 2 \\
\hline ARIEGE & 09 & af. Estatz (af. Ariège) & * & $42^{\circ} 40$ & 1800 & $\mathrm{R}$ & $1-2$ & pel. subalpine & 07 & 1 \\
\hline CATALOG & & dév. lac Montolieu (af. Ynola) & & $42^{\circ} 40$ & 2000 & $\mathrm{R}$ & $2-4$ & pel. subalpine & 09 & 1 \\
\hline HAU-GAR & 31 & af. Pique (< Port Venasque) & * & $42^{\circ} 40$ & 2100 & $\mathrm{R}$ & $1-2$ & pel. alpine & 06 à 10 & 2 \\
\hline ARAGON & & af. Esera (Aneto) & * & $42^{\circ} 40$ & 2400 & M & $1-2$ & pel. alpine & 06 à 10 & 2 \\
\hline ARAGON & & af. Bsera (< Port Venasque) & * & $42^{\circ} 40$ & 2100 & L & $1-3$ & pel. alpine & 06 à 10 & 2 \\
\hline ARAGON & & af. rio Gallego & & $42^{\circ} 50$ & 1760 & $\mathbb{R}$ & $2-4$ & pel. subalpine & 05 à 11 & 6 \\
\hline ARAGON & & rio Gallego & & $42^{\circ} 50$ & 1750 & H & $1-3$ & pel. subalpine & 05 à 11 & 6 \\
\hline PYR-ATL & 64 & Glère, af. Brousset (af. Ossau) & & $42^{\circ} 50$ & 1980 & $\mathbb{R}$ & $1-2$ & pel. subalpine & 07 à 11 & 3 \\
\hline PYR-ATL & 64 & af. Bious (af. Ossau) & * & $42^{\circ} 50$ & $1590^{\circ}$ & M & $1-2$ & pel. montagnarde & 03 à 11 & 7 \\
\hline PYR-ATL & 64 & Iscoo, af. Valentin (af. Ossau) & * & $43^{\circ}$ & 850 & $R$ & $1-3$ & hêtraie montagnarde & 03 à 11 & 7 \\
\hline PYR-ATL & 64 & Serres (af. Ossau) & & $43^{\circ}$ & 830 & II & $1-3$ & pel, collinéenne & 03 à 11 & 7 \\
\hline PYR-ATL & 64 & r. au nord de Béon (af. Ossau) & * & $43^{\circ}$ & 450 & M & $1-3$ & feuillus & 03 à 11 & 7 \\
\hline PYR-AML & 64 & af. Olhadoko (af. Larrau) & * & $43^{\circ}$ & 1300 & $\mathrm{R}$ & $1-2$ & hêtraie montagnarde & 07 à 08 & 2 \\
\hline NAVARRE & & rio Urrobi (> Roncesvalles) & & $43^{\circ}$ & 1000 & $\mathrm{R}$ & $2-4$ & forêt de feuillus & 05 à 08 & 3 \\
\hline
\end{tabular}

LARG : largeur du lit, $\mathrm{N}:$ nombre de prélèvements, $\mathrm{r}$. : ruisseau, af. : affluent, dév. : déversoir, pel. : pelouse LOC : Localité, $N^{\circ}$ du département, CRE : crénon, LATI : latitude, ALTI : Altitude, VIC : vitesse moyenne du courant, $\mathbb{R}$ : très rapide, $R:$ rapide, $M:$ modéré, $L:$ lent

PYR-ORI : Pyrénées-Orientales, HAU-GAR : Haute-Garonne, PYR-ATL : Pyrénées-Atlantiques, CATALOG : Catalogne 
Tableau 3. Caractéristiques des stations prospectées dans les sierras de la Péninsule Ibérique.

Table 3. Characteristics of the sampling stations in the Iberic Sierras.

\begin{tabular}{|c|c|c|c|c|c|c|c|c|}
\hline COURS D'EAU & CRE & LATI & ALPI & WIC & LARG & VEGETATION BORDANTE & MOIS & $\mathbb{N}$ \\
\hline \multicolumn{9}{|l|}{ CANTABRIQUE (Cantabr. Fig 1) } \\
\hline rio Miera (Portillo de Lunada) & & $43^{\circ} 10$ & 600 & $\mathrm{R}$ & $6-9$ & feuillus & 07 & 1 \\
\hline af. Deva (Puerto de San Glorio) & * & $43^{\circ}$ & 1600 & $R$ & $2-4$ & pel. montagn. buissons & 08 & 1 \\
\hline fuente Dé, af. Deva (P. de Europa) & * & $43^{\circ} 10$ & 1100 & II & $2-4$ & feuillus & 08 & 1 \\
\hline af. Cares (Puerto de Pandetrave) & & $43^{\circ} 10$ & 1400 & $\mathrm{R}$ & $4-6$ & feuillus & 08 & 1 \\
\hline r. Casano (Picos de Furopa) & * & $43^{\circ} 20$ & 1300 & L & $1-3$ & pel. montagnarde & DB & 1 \\
\hline < col Mazada (Picos de Buropa) & * & $43^{\circ} 10$ & 2000 & M & $1-2$ & pel. subalpine & 08 & 1 \\
\hline Junjumia, af. Sella (P. Europa) & * & $43^{\circ} 10$ & 1500 & H & $1-2$ & pel. montagnarde & OB & 1 \\
\hline af. Huerna > Tuiza (Pena Obina) & $t$ & $43^{\circ}$ & 1600 & a & $1-2$ & pel. Lontagnarde & 08 & 1 \\
\hline \multicolumn{9}{|l|}{ CABRRRA (Cab. Fig 1) } \\
\hline El Puente (Sanabria) & & $42^{\circ} 10$ & & $M$ & $2-3$ & feuillus & 05 & 1 \\
\hline \multicolumn{9}{|l|}{ DEMANDA (Dem. Pig 1) } \\
\hline af. Urbion (nord pic Trigaza) & * & $42^{\circ} 20$ & 1800 & $\mathbf{R}$ & $2-4$ & pel. montagn. buissons & 08 & 1 \\
\hline af. 0ja (< station Valdezcaray) & & $42^{\circ} 20$ & 1500 & $\mathbf{R}$ & $2-4$ & pel. montagnarde & 05 à 08 & 2 \\
\hline af. 0ja (> station Valdezcaray) & * & $42^{\circ} 20$ & 2000 & $y$ & $1-2$ & pel. subalpine & 05 à 08 & 2 \\
\hline \multicolumn{9}{|l|}{ GUDAR ET JAVALAVBRE } \\
\hline r. Alcala, af. Valbona, Virgen Vega & & $40^{\circ} 20$ & 1400 & $\mathrm{~L}$ & $4-6$ & pel. + feuillus & 05 à 07 & 2 \\
\hline r. Camarena (Sierra de Javalamil & & $40^{\circ} 10$ & 1300 & R & $3-5$ & fevillus & 05 à 07 & 2 \\
\hline \multicolumn{9}{|l|}{ GUADARRAMA (Guad. Fig 1) } \\
\hline af. Lozoya (station de Valdesqui) & $*$ & $41^{\circ} 10$ & 2000 & $\mathbf{R}$ & $1-2$ & pel. subalpine & 07 & 1 \\
\hline \multicolumn{9}{|l|}{ CANDEIARIO (Cand. Fig 1) } \\
\hline Candelario (route de la Laguna) & * & $40^{\circ} 20$ & 1800 & TR & $1-2$ & pel. rontagnarde & 05 à 07 & 2 \\
\hline Cuerpo de Hoabre (Candelario) & & $40^{\circ} 20$ & 1300 & $\mathbb{R}$ & $2-3$ & hêtraie montagnarde & 05 à 07 & 2 \\
\hline \multicolumn{9}{|l|}{ MAYEDE (Man. Fig 1) } \\
\hline r. versant nord, serra de Mamede & & $39^{\circ} 20$ & 700 & $\mathbf{R}$ & $1-2$ & pel. + feuillus & 07 & 1 \\
\hline \multicolumn{9}{|l|}{ SAGRA (S. Fig 1) } \\
\hline af. Bravatas & * & $38^{\circ}$ & 1450 & M & $1-2$ & broussailles & 07 & 1 \\
\hline r. Raigadas (cortijo de la Losa) & * & $38^{\circ}$ & 1500 & $\mathrm{R}$ & $1-2$ & paturages + fevillus & 07 & 1 \\
\hline \multicolumn{9}{|l|}{ CAZORLA (Caz. Fig 1) } \\
\hline$<$ pic Cabanas & * & $37^{\circ} 50$ & 1700 & $\mathbf{R}$ & $1-2$ & & 04 & 1 \\
\hline \multicolumn{9}{|l|}{ ALAADEN (AI. Fig 1) } \\
\hline Puenrayor (sud de Torres) & * & $37^{\circ} 50$ & 1600 & $\mathbf{R}$ & $1-2$ & pel. montagn. feuillus & 04 à 07 & 2 \\
\hline \multicolumn{9}{|l|}{ FILABRRS (F. Pig 1) } \\
\hline ( Calar Alto (versant nord) & * & $37^{\circ} 10$ & 1800 & M & $1-2$ & feuillus & 04 & 1 \\
\hline \multicolumn{9}{|l|}{ NEVADA (N. Pig 1) } \\
\hline af. rio Grande (Puerto de la Raqua) & * & $37^{\circ} 10$ & 2000 & II & $1-2$ & pel. subalpine & 04 à 07 & 2 \\
\hline r. Trevelez & & $37^{\circ}$ & 1500 & $\mathbb{T}$ & $4-8$ & pel. + fevillus & 07 & \\
\hline af. r. Kulhacen & $*$ & $37^{\circ}$ & 3000 & $\mathbf{R}$ & $1-2$ & pel. alpine & 07 & 1 \\
\hline r. Lanjaron à Lanjaron & & $37^{\circ}$ & 660 & $\mathbb{T R}$ & $4-6$ & pel. + fevillus & 04 & 1 \\
\hline r. Monachil & * & $37^{\circ}$ & 2700 & $\mathbf{R}$ & $1-2$ & pel. alpine & 07 & 1 \\
\hline r. Monachil & & $37^{\circ}$ & 2000 & $\mathrm{R}$ & $4-6$ & pel. subalpine & 04 à 07 & 2 \\
\hline \multicolumn{9}{|l|}{ TRJEDA (Tej. Pig 1) } \\
\hline$>$ cortijo Huerta de Hoyas & * & $36^{\circ} 50$ & 1200 & H & $1-2$ & broussailles & 04 à 07 & 2 \\
\hline \multicolumn{9}{|l|}{ HOHCHIQUE (Mon. Pig 1) } \\
\hline Caldaas de Monchique & & $37^{\circ} 20$ & 500 & $\mathbf{R}$ & $1-2$ & feuillus & 07 & 1 \\
\hline < barrage, (versant est) & & $37^{\circ} 20$ & 700 & H & $1-2$ & feuillus & 07 & 1 \\
\hline
\end{tabular}

LOC : Localité, CRR : crenon, LATI : latitude, ALPI : Altitude, WMC : vitesse moyenne du courant, LARG : largeur du lit, N : noabre de prélèvenents, r. : ruisseau, af. : affluent, f. : fuente, dév. : déversoir, pel. : pelouse $\mathbb{R}$ : très rapide, $R:$ rapide, $M:$ nodéré, $L$ : lent 
Tableau 4. Espèces recensées dans les stations prospectées. Table 4. List of the species collected.

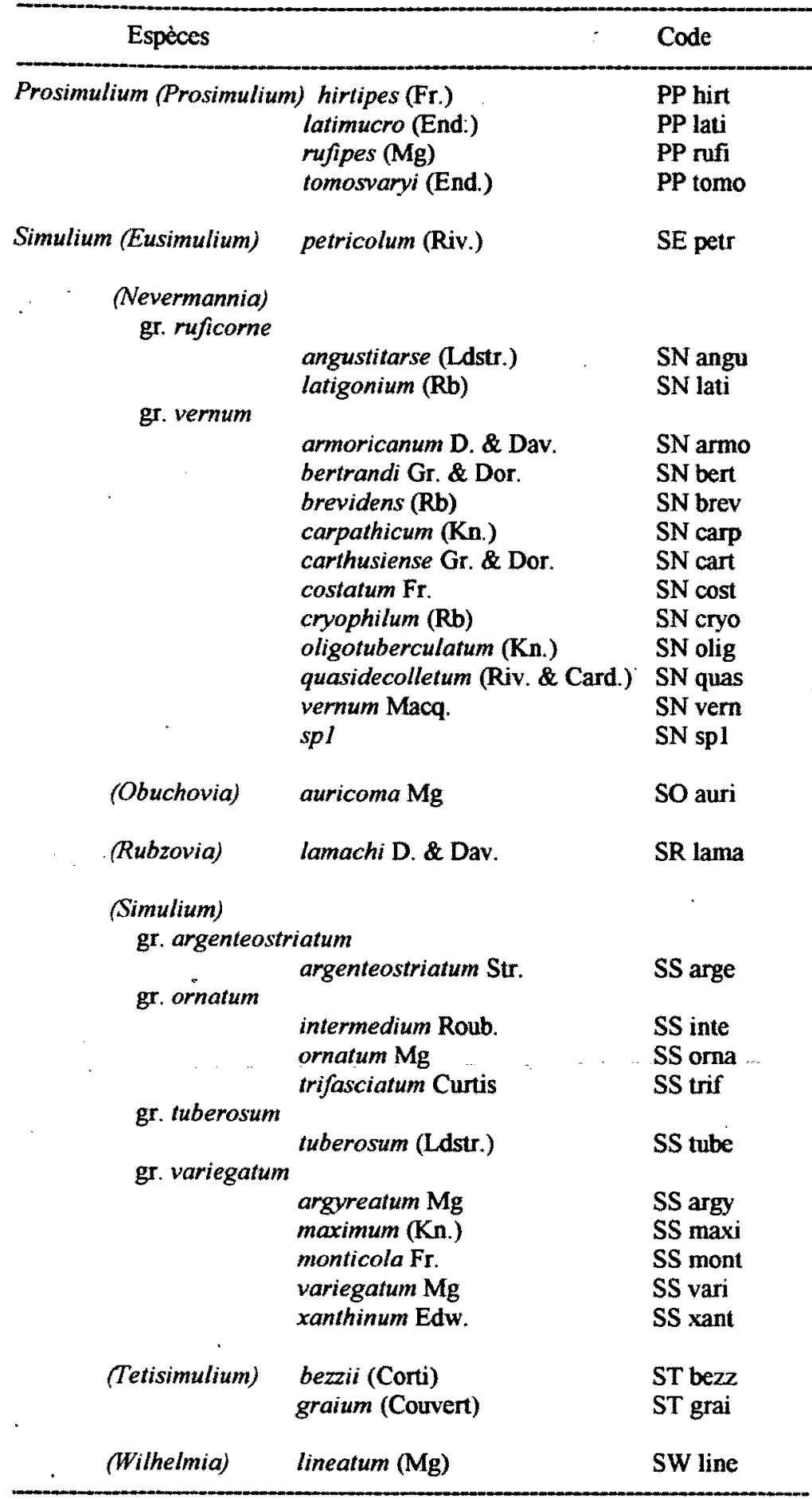

$S$. (N.) oligotuberculatum n'était signalée que des Monts Tatras et Jeseniky à l'est de la Tchécoslovaquie (Knoz 1965). Nous l'avons retrouvée dans plusieurs stations des Alpes françaises et italiennes, en très haute altitude, le plus souvent au-dessus de $2000 \mathrm{~m}$.

$S$. (T.) graium $=S$. (T.) ? bezzii. Cette espèce, décrite par Couvert (1967) et placée récemment en équivalence avec $S$. (T.) bezzi sous forme interrogative, par Crosskey (1988), se développe au-dessus de $2000 \mathrm{~m}$ d'altitude, dans une zone très restreinte de la chaîne alpine, sur les versants italiens et français du col du Petit-Saint-Bernard.

\section{b) Espèces ibériques}

$S$. (R.) lamachi, signalée d'Espagne (ClergueGazeau \& Vinçon 1990) est une forme crénophile rare (biotope de faible luminosité) qui vit dans quelques gîtes très localisés sur le pourtour ouest de la Méditerranée.

$S$. (N.) sp. 1. Certaines larves et nymphes prélevées dans la Sierra de Gudar, à l'est de Teruel, semblent appartenir à une espèce encore inconnue. Seule la récole d'adultes mâles et femelles permettra de le confirmer et d'établir éventuellement la diagnose de cette espèce. Elle a été récoltée à $1400 \mathrm{~m}$ d'altitude dans un ruisseau de faible largeur $(1 \mathrm{~m}$ environ), de courant lent, et dans lequel se développe une abondante population de macrophytes aquatiques.

\section{Les Simulies du milieu lotique monta- gnard : crénal et épirhithral}

\subsection{Preferendum altitudinal et température de l'eau} (Fig. 2)

a) Espèces sténothermes d'eau froide (ruisseaux de haute altitude)

$S$. (N.) carpathicum, $P$. (P.) latimucro, $S$. (N.) oligotuberculatum, $S$. (N.) vernum, $S$. (N.) brevidens, $S$. (N.) carthusiense, $S$. (S.) maximum, $S$. (N.) bertrandi, $S$. (E.) petricolum et $S$. (N.) quasidecolletum sont les espèces les plus strictement inféodées aux ruisseaux de haute montagne (1400-2800 m, épirhithral et crénal) ce qui met en évidence leur caractère sténotherme d'eau froide.

Trois d'entre elles ont une préférence marquée pour les sources et les cours d'eau de très haute montagne situés au-dessus de $2000 \mathrm{~m}$ d'altitude : $P$. $(P$.) latimucro, $S$. (N.) carpathicum et $S$. (N.) oligotuberculatum.

$P$. (P.) latimucro, la plus largement répartie sur la zone considérée, est très fréquente et assez abondante dans tous les principaux massifs sudoccidentaux.

$S$. (N.) carpathicum semble ne pas se développer en Espagne sauf dans la chaîne pyrénéenne où elle est fréquente et abondante ; dans les Alpes, elle paraît très localisée. 
Tableau 5. Répartition des espèces, en classes d'abondance, dans les cours d'eau des Alpes occidentales, du nord au sud. Table 5. Species distribution in the occidental Alps stations, from north to south.

PP PP PP PP SE SN SN SN SN SN SN SN SN SN SO SS SS SS SS SS SS SS SS SP ST $\mathrm{c} d$. hi la ru to pe be br ca ca co cr ol qu ve au ar in or tr ar ma mo va be gr sp It ti fi mo tr rt ev rp rt st yo ig as $\mathrm{rn}$ ri ge te na if gy xi nt ri $z z$ ai

\begin{tabular}{|c|c|c|c|c|c|c|c|c|c|c|c|c|c|c|c|c|c|c|}
\hline 73 & af. Reclus & 2100 & & & 4 & 1 & & 2 & & 3 & & & & & 2 & & & $4 \div 7$ \\
\hline 73 & Ste Foy Taren. & 1000 & & & 4 & & & 1 & & 1 & 2 & & & 4 & 2 & 41 & & \\
\hline 73 & Youlins & 1400 & & & 2 & 2 & 2 & 1 & & 1 & & & & 5 & 2 & 1 & & \\
\hline 73 & Lenta & 2300 & & 1 & 2 & & & & & 1 & & & & & & & & $*$ \\
\hline 38 & Vence & 1000 & 1 & 2 & 2 & 2 & 2 & 1 & & 1 & 4 & & 1 & 1 & & & & 12 \\
\hline 38 & Vence & 800 & & & & & & & & & & 1 & & 1 & & & & \\
\hline 38 & Vence & 240 & 1 & & & & & & & & & & & 2 & & 2 & & \\
\hline 38 & La Grange & 1000 & & 2 & 2 & 2 & 3 & & 3 & 4 & & & & 2 & & 1 & & \\
\hline 38 & Rif Garcin & 2030 & & 2 & & & & & & & 3 & & & & & & & $*$ \\
\hline 38 & af. Flumet & 1900 & 2 & 2 & 2 & & & & & 2 & & & 1 & & & & & \\
\hline 38 & Drevenne & 1000 & & & 2 & & 3 & & & & 4 & & & & & 2 & & $\star$ \\
\hline 38 & Drevenne & 700 & 3 & & 3 & & & & & & & & & 1 & 1 & & & \\
\hline 38 & Voroize & 600 & & & & & & & 3 & & & & & & & & & * \\
\hline 38 & Furon & 1300 & 3 & & & & 1 & & & & 1 & & & 2 & & & & $\star$ \\
\hline 38 & Pellas & 1400 & & & & 1 & 1 & & & & & & & & & & & $*$ \\
\hline 38 & Pellas & 1200 & 3 & 2 & 2 & & & 4 & & & & & & & & & & \\
\hline 38 & af. Bbron & 1100 & 4 & & 2 & 1 & & & & & & & & & & & & \\
\hline 38 & Jocou & 1500 & & & & & & & & 1 & 1 & & & & & & & * \\
\hline 26 & Archiane & 1300 & & & & 2 & 2 & 3 & & 2 & & & & & & 3 & & \\
\hline 26 & Archiane & 600 & 1 & & & & & & & & & 1 & & 2 & & 3 & & \\
\hline 05 & Bléronnet & 1800 & & & 3 & & & 4 & & & & & & & 1 & & - & \\
\hline 05 & Bouchet & 2300 & & 1 & 3 & & 4 & & & 12 & & & & & 2 & & & $*$ \\
\hline 05 & af. Bouchouse & 2700 & & & 1 & & & 32 & & 1 & 2 & & & & 2 & & & $*$ \\
\hline 05 & Faito & 2400 & & & & & & 22 & & 21 & & & & & & & & * \\
\hline 05 & Aigue Agnelle & 2600 & & & & & & & & 2 & & & & & & & & $\star$ \\
\hline 05 & af. Chagne & 2100 & & 2 & & & 1 & & & 21 & & & & & & & & * \\
\hline 04 & af. Mounal & 2000 & & 1 & & & & & & 1 & & & & & & & & \\
\hline 14 & Oronaye & 2000 & & 2 & 3 & 1 & & & & 2 & 1 & & & & & & & \\
\hline 04 & af. Bachelard & 2200 & & 1 & 3 & & & & & 2 & & & & & 1 & & & * \\
\hline 04 & af. Bachelard & 1800 & 2 & & 1 & & 1 & 3 & & 1 & & & & & 1 & & 3 & \\
\hline 06 & Tinée & 2300 & & 2 & 4 & 1 & 12 & & & 21 & & & & & 2 & & & $*$ \\
\hline 06 & af. Tinée & 2000 & & & 3 & 1 & 1 & 2 & & 2 & & & & & & & & \\
\hline 06 & af. Vésubie . & 1500 & & & & 2 & 2 & & & 2 & & & & & & & & $*$ \\
\hline 06 & Sce af. Bévéra & 1700 & & & & & & 4 & & & 4 & & & 3 & & & & $*$ \\
\hline 06 & Refrei & 1900 & & & 3 & 1 & 1 & & 4 & $4 \cdot 2$ & & & & 2 & 2 & & & * \\
\hline 06 & Caramagne & 1400 & & & & 2 & 22 & & & 2 & & & & 2 & & & & \\
\hline 06 & Roya & 850 & & & & & & & 2 & 2 & & & & 2 & & 2 & & \\
\hline ITA & af. Sermenza & 1400 & & 1 & & 2 & 2 & & 2 & 1 & & & & & & & & \\
\hline ITA & S. di denonte & 1900 & & & 1 & & & & 3 & 32 & & & 1 & 1 & & & 3 & \\
\hline ITA & P. del. Chiesa & 1900 & & & & & 2 & & & 1 & & & & & & & & \\
\hline ITA & af. P. Chiesa & 2000 & & & & 4 & 42 & & 3 & 32 & 2 & & & 2 & 3 & & & * \\
\hline ITA & af. P. Chiesa & 1600 & & & 1 & & & & & 1 & & & & 3 & 1 & & & \\
\hline
\end{tabular}

Fréq. crénal 19 st. $\quad \begin{array}{llllllllllllllllllllllllll}5 & 32 & 42 & 0 & 5 & 26 & 32 & 11 & 21 & 5 & 47 & 58 & 16 & 21 & 0 & 0 & 0 & 0 & 5 & 16 & 37 & 5 & 0 & 0 & 5\end{array}$ Fréq. épirh. 23 st. $\quad \begin{array}{llllllllllllllllllllllllll}35 & 30 & 61 & 17 & 0 & 26 & 17 & 4 & 35 & 4 & 52 & 30 & 4 & 9 & 4 & 4 & 4 & 13 & 4 & 48 & 26 & 22 & 17 & 9 & 0\end{array}$ Fréq. c. +é. 42 st. $\quad \begin{array}{llllllllllllllllllllllllll}21 & 31 & 52 & 10 & 2 & 26 & 24 & 7 & 29 & 5 & 50 & 43 & 10 & 14 & 2 & 2 & 2 & 7 & 5 & 33 & 31 & 14 & 10 & 5 & 2\end{array}$

ITA : Italie, d. sp : diversité spécifique, c : crénal, é : épirnithral, st. : stations

Classes d'abondances : 1 (1 à 3 individus), 2 (4 à 10), 3 (11 à 30), 4 (31 à 100), 5 (> à 100) 
Tableau 6. Répartition des espèces dans les cours d'eau des Pyrénées, (d'est en ouest).

Table 6. Species distribution in the Pyrenean stations, from east to west.

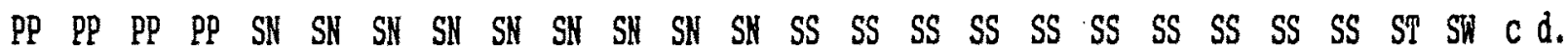
hi la ru to an la be br ca ca cr qu ve ar in or tr tu ar ma ma xa be li sp rt ti fi mo gu ti rt ev rp rt yo as $\mathrm{rn}$ ge te na if be gy xi nt ri nt z2 ne

\begin{tabular}{|c|c|c|c|c|c|c|c|c|c|c|c|c|c|c|c|c|c|c|c|c|}
\hline 66 & Llitéra & 2200 & 2 & & & & & & & & & & & & & & & & & t \\
\hline 66 & Riberole & 2400 & 1 & & & & & & & & & & & & & & & & & * \\
\hline 66 & Eyne & 2500 & 2 & & & & & & & & & & & & & & & & & * \\
\hline 66 & Err & 2200 & & & & & 1 & & & & & & & & & & & & & \\
\hline 66 & Err & 1900 & & & & & & & & & & & & & 1 & & & & & \\
\hline 11 & af. Aiguette & 2300 & 1 & & & & & 2 & & & 2 & & & & & & & & & \\
\hline 66 & af. Grave & 2425 & & & & & 2 & 2 & & & & & & & & & & & & \\
\hline 66 & Fourats & 2400 & & & & & & 3 & & & & & & & & & & & & \\
\hline 66 & M. d'Eygues & 2350 & 3 & & & & 3 & 1 & & & 3 & & & & & & & & & \\
\hline 66 & C. Rousseau & 2400 & 2 & & & & 4 & 2 & & & 2 & & & & & & & & & \\
\hline 66 & C. Rousseau & 2200 & & & & & 3 & & & & & & & & & & & & & \\
\hline 66 . & En Garcia & 2300 & 2 & & & & 2 & 2 & 1 & & 2 & & & & & & & & & \\
\hline 66 & En Garcia & 2000 & & & & & & & 1 & & 2 & & & & & & & & & \\
\hline CAT & r. > Estana & 1800 & & & & 2 & & & & & & & & & 1 & & & & & \\
\hline CAT & r. > Estana & 1600 & & & & & & & 2 & & ? & & & & 1 & 1 & 3 & & & \\
\hline CAT & Duran & 2500 & & & & & & & & & 2 & & & & & & & & & \\
\hline $\begin{array}{l}\text { CAT } \\
\text { CAT }\end{array}$ & $\begin{array}{l}\text { Duran } \\
\text { p. de Tosas }\end{array}$ & $\begin{array}{l}2300 \\
1700\end{array}$ & 4 & 3 & & & 8 & & 2 & & & & & & 1 & & & & & \\
\hline 09 & af. Ariège & 2200 & & & & & 2 & 2 & 1 & & 2 & & & & & & & & & \\
\hline 09 & af. Ariège & 1900 & 3 & & & & & 2 & 1 & & & & & & & 2 & & & & \\
\hline 09 & Sant Josep & 2000 & 1 & & & & & 2. & & & & & & & & & & & & \\
\hline AND & Tristaina & 2200 & 2 & 1 & & & & & & & & & & & & 1 & & & & \\
\hline AND & Coma Pedrosa & 2700 & -2 & & & & & . & & & & & & & & & & & & \\
\hline AND & Coma Pedrosa & 2550 & 2 & & & & & & & & 2 & & & & & & & & & \\
\hline AND & Coma Pedrosa & 2400 & & & & & & & 1 & & & & & & & & & & & \\
\hline 09 & af. Estatz & 1800 & & 1 & & & & 1 & & & & & & & & & & & & \\
\hline CAT & d. Montolieu & 2000 & & & & & & & & & 1 & & & & 1 & & & & 2 & \\
\hline 31 & af. Pique & 2100 & & & & & 1 & & & & & & & & & & & & & \\
\hline ARA & af. Esera & 2400 & 1 & & & & 3 & & & & 1 & & & & & & & & & \\
\hline ARA & af. Bsera & 2100 & 3 & & & & & & & & & & & & & & & & & * \\
\hline ARA & af. Gallego & 1760 & 1 & 2 & & & & 3 & 3 & & & & & & 2 & 1 & 3 & 3 & 3 & \\
\hline ARA & rio Gallego & 1750 & 3 & 3 & & & 1 & 3 & & 1 & & 1 & 3 & & 2 & 1 & & 3 & 4 & \\
\hline 64 & Glère & 1980 & 2 & 4 & & 2 & & 3 & & & & & & & 2 & & 1 & & 1 & \\
\hline 64 & af. Bious & 1590 & & 2 & & & 1 & & & & & & & & & & & & & $t$ \\
\hline 64 & Iscoo & 850 & 1 & 3 & & & & & & & & & & & 2 & & & & & * \\
\hline 64 & Serres & 830 & & & 2 & 4 & & 2 & 2 & & 1 & 44 & 3 & 2 & 3 & & 3 & 3 & 1 & 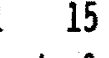 \\
\hline 64 & r. de Béon & 450 & & 2 & & & & 3 & 4 & & & 44 & & & 2 & & 3 & 3 & 1 & $\star$ \\
\hline 64 & af. Olhadoko & 1300 & & & & & & 1 & 2 & & & & & & & & & & & $*$ \\
\hline NAV - & rio Urrobi & 1000 & & & & & & & & & & & & & & & 3 & & & \\
\hline
\end{tabular}

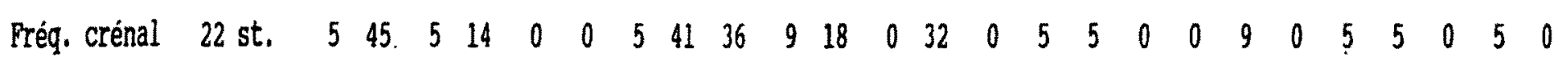
Fréq. épirh. 17 st. 2918 29 18 0 $6 \begin{array}{lllllllllllllllllllll}6 & 6 & 6 & 18 & 12 & 24 & 41 & 6 & 24 & 6 & 12 & 6 & 12 & 6 & 47 & 29 & 29 & 18 & 6 & 29 & 6\end{array}$

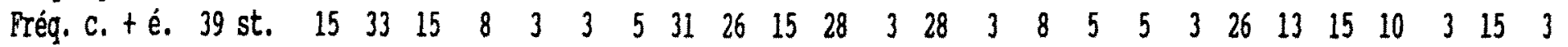

CAT : Catalogne, AND : Andorre, ARA : Aragon, NaV : Navarre, d. sp : diversité spécifique, c : crénal, é : épirhithral, st. : stations, Classes d'abondances : 1 (1 à 3 individus), 2 (4 à 10), 3 (11 à 30), 4 (31 à 100), 5 (> à 100) 
Tableau 7. Répartition des espèces dans les cours d'eau de la Péninsule Ibérique.

Table 7. Species distribution in the Iberic stations.

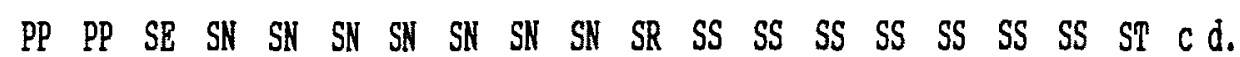

la to pe ar br ca co cr ve sp la in or tr ar ma mo va be sp

ti $\mathbb{m o}_{0}$ tr mo ev rt st yo $\mathrm{rn} 1$ ma te na if gy xi nt ri 22

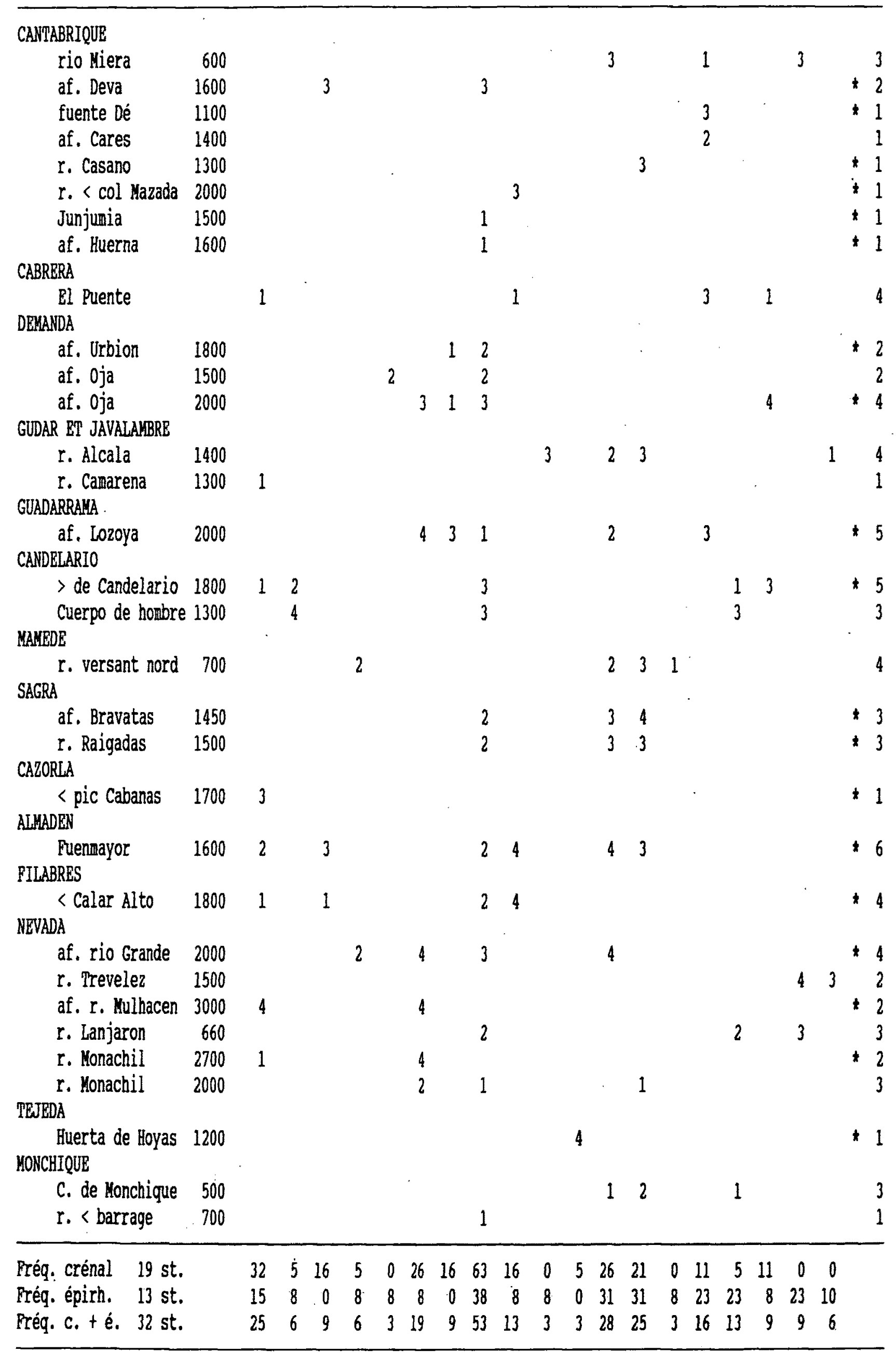




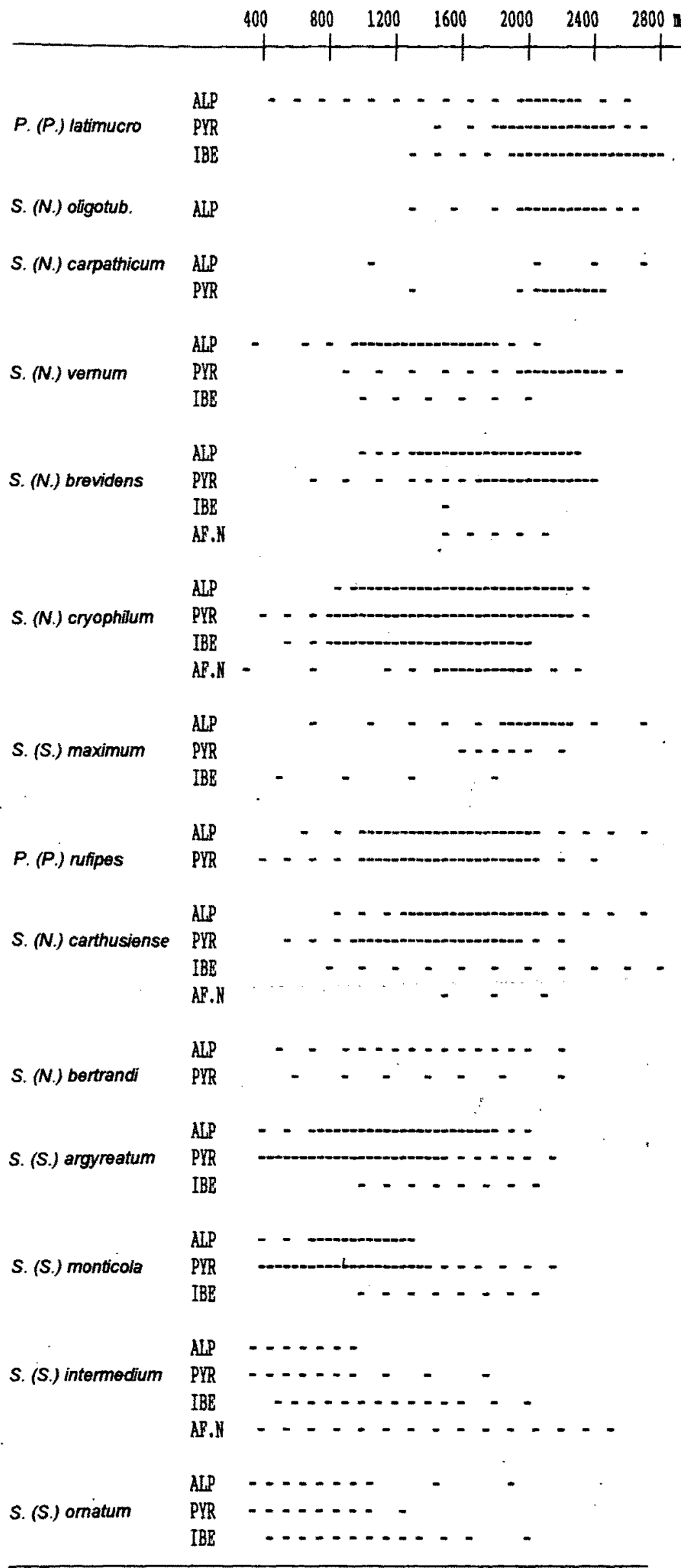

ALP : Alpes, PYR : Pyrénées, IBE : péninsule ibérique, AF.I : Afrique du Nord

Fig. 2. Répartition altitudinale des espèces les plus fréquentes dans le crénal et l'épirhithral du milieu lotique montagnard.

Fig. 2. Altitudinal distribution of the species living in the crenal and epirhithral zones.
$S$. (N.) oligotuberculatum, connue uniquement de la région alpine, y est fréquente mais peu abondante (Tableau 5).

Ces 3 espèces peuvent se développer à des altitudes extrêmes : $3000 \mathrm{~m}$ pour $P$. (P.) latimucro dans la Sierra Nevada, $2700 \mathrm{~m}$ pour $S$. (N.) carpathicum et $S$. (N.) oligotuberculatum dans le massif du Queyras (Hautes-Alpes). En revanche, on ne les rencontre que très exceptionnellement en moyenne montagne.

$S$. (N.) vernum, $S$. (N.) brevidens, $S$. (N.) carthusiense, $S$. (N.) bertrandi et $S$. (S.) maximum peuvent se développer à des altitudes. un peu plus basses. Elles sont cependant rares au-dessous de $1000 \mathrm{~m}$ et on les rencontre alors principalement au voisinage des sources où elles retrouvent des conditions thermiques propices à leur développement. Parmi cellesci, $S$. (N.) vernum et $S$. (N.) brevidens sont les plus crénophiles : $S$. (N.) carthusiense, $S$. (S.) maximum et $S$. (N.) bertrandi, à valence écologique assez large, se développent aussi bien dans les ruisseaux de sources que dans les torrents froids de montagne.

$S$. (E.) petricolum et $S$. (N.) quasidecolletum sont des formes alticoles « relativement 》 rares ; la première a été rencontrée dans 1 station alpine, à $2100 \mathrm{~m}$ d'altitude et dans 3 stations espagnoles, entre 1600 et $1800 \mathrm{~m}$. Comme pour les stations des Pyrénées (Lavandier 1976) et d'Afrique du Nord, il s'agit de ruisseaux et ruisselets émissaires de sources de haute montagne. $S$. (N.) quasidecolletum, signalée précédemment dans les Pyrénées espagnoles, sous le nom de $S$. (N.) carthusiense ? truncatum (Vinçon et Clergue-Gazeau 1988), a été localisée dans 4 stations alpines, sur les versants français et italien ; elle se développe dans des ruisselets de sources, entre 1000 et $2700 \mathrm{~m}$ d'altitude.

\section{b) Espèces montagnardes plus eurythermes}

Dans ce groupe, nous classons des espèces plus eurythermes dont la répartition est plus ou moins étendue en moyenne et haute montagne. $S$. (N.) cryophilum et $P$. (P.) rufipes sont les plus alticoles et possèdent une large valence écologique ; elles colonisent le crénal et l'ensemble du rhithral.

$P$. (P.) hirtipes, $S$. (S.) argyreatum, $S$. (S.) monticola, $S$. (S.) variegatum, $S$. (T.) bezzii, $S$. (S.) intermedium et $S$. (S.) ornatum sont plus caractéristiques des cours d'eau de moyenne montagne et de piémont. 
$S$. (N.) costatum, S. (R.) lamachi et $P$. (P.) tomosvaryi se développent dans les ruisseaux de colline ou de piémont et ont une tendance nettement plus crénophile que les espèces précédemment citées.

\subsection{Préférendum altitudinal et influence de la latitude}

La comparaison des peuplements des zones montagneuses étudiées, confrontée à des observations provenant d'Afrique du Nord (Clergue-Gazeau et al. 1991), montre l'influence de la situation géographique (latitude des stations) sur la répartition altitudinale de certaines espèces (Fig .2).

\section{S. (N.) costatum}

Dans les Alpes, cette espèce crénophile vit en moyenne montagne ; en Espagne, elle se développe jusqu'à $2000 \mathrm{~m}$ dans les Sierras de la Demanda et de Guadarrama (centre Espagne) ; dans le HautAtlas marocain, elle dépasse 2800 m (Bouzidi 1989, Clergue-Gazeau \& al. 1991). Elle n'a encore jamais été rencontrée dans les Pyrénées, malgré les nombreuses prospections réalisées.

\section{S. (S.) intermedium}

Dans les Alpes, elle colonise principalement les ruisseaux et torrents de piémont (au-dessous de $1000 \mathrm{~m}$ ) ; dans les Pyrénées, elle peuple les ruisseaux de basse et moyenne montagne (exceptionnellement jusqu'à $1800 \mathrm{~m}$ ). Dans les Sierras de Guadarrama (centre de l'Espagne) et Nevada (Andalousie), elle atteint $2000 \mathrm{~m}$ et dépasse $2500 \mathrm{~m}$ dans le Haut-Atlas, marocain (Bouzidi 1989, ClergueGazeau \& al. 1991).

Les sources du Haut-Atlas ou des sierras andalouses, au-dessus de $2000 \mathrm{~m}$, présentent des caractéristiques écologiques et notamment des conditions thermiques qui se rapprochent de celles des biotopes de moyenne montagne dans les Alpes ou les Pyrénées, cela explique les décalages observés dans la répartition altitudinale des espèces (Giudicelli \& Dakki 1984, Giudicelli \& Thierry 1985).

De tels décalages ont été observés pour d'autres groupes d'insectes aquatiques et notamment pour les Plécoptères suivants : Dinocras cephalotes, Perla marginata, Protonemura meyeri, Capnioneura mitis et Leuctra fusca (Aubert 1956 et 1986, Berthélemy 1966, Sanchez-Ortega \& Alba-Tercedor 1990, Vinçon 1987 a et b). Ainsi, dans les Alpes, Perla marginata colonise les cours d'eau de plaine ou de piémont et dépasse rarement $800 \mathrm{~m}$; dans les Pyrénées, elle se développe en basse et moyenne montagne $(300 \mathrm{~m}$ et $1200 \mathrm{~m})$, exceptionnellement au-dessus dans des déversoirs de lacs. Elle atteint $2500 \mathrm{~m}$ dans la Sierra Nevada et $2900 \mathrm{~m}$ dans le Haut-Atlas marocain.

\section{Conclusion}

Cette étude menée dans différents massifs montagneux du Sud-Ouest de l'Europe et notamment en haute altitude, a permis de mieux connaître la faune simuliidienne des sources et de leurs ruisseaux émissaires, et d'apprécier l'abondance et la diversité des peuplements dans ces biotopes soumis à des conditions de milieu difficiles, voire extrêmes.

Des populations de Simulies ont été observées dans plusieurs stations de très haute altitude audessus de $2500 \mathrm{~m}$, dans les Alpes, les Pyrénées et la Sierra Nevada : $S$. (N.) carpathicum dans 8 stations entre 2300 et $2700 \mathrm{~m}$ (moyenne $2130 \mathrm{~m}$ ), $P$. (P.) latimucro dans 15 stations entre 2300 et $3000 \mathrm{~m}$ (moyenne $2060 \mathrm{~m}$ ) et $S$. (N.) oligotuberculatum dans 6 stations alpines entre 2300 et $2700 \mathrm{~m}$ (moyenne $2020 \mathrm{~m}$ ). Ces 3 espèces très alticoles apparaissent comme une composante importante du peuplement benthique de certains biotopes de haute montagne, pauvres en matière organique et de faible richesse spécifique.

La prospection de biotopes difficiles d'accès à permis de mieux connaître la répartition et l'écologie de certaines espèces rares ou localisées.

L'examen d'un grand nombre d'individus récoltés à tous les stades de développement dans de nombreuses stations (114) et l'observation des paramètres du milieu, mettent en relief le rôle prédominant de l'altitude et de la température dans la distribution spatiale des espèces et dans la composition des peuplements.

\section{Travaux cités}

Aubert J. 1956. - Contribution à l'étude des Plécoptères d'Afrique du Nord. Mitt. Schweiz. ent. Ges., 29 (4) : 419-436.

Aubert J. 1963. - Les Plécoptères de la Péninsule Ibérique. Eos, Madr., $39: 23-107$.

Aubert J. 1986. - Les Plécoptères des Alpes françaises. Annls Soc. ent. Fr. (N.S.), 22 (1) : 81-104.

Beaucournu-Saguez F. 1972. - Captures de Simulies dans la moitié sud du Portugal. Premières captures en Europe de Simulium sergenti Edwards 1923 et de Simulium ruficorne Macquart 1838. An. Esc. nac. Saude publ. Med. trop., $6: 73-83$.

Beaucournu-Saguez F. 1975a. - Sur quelques Simulies (Diptera, Simuliidae) du Nord-Ouest de l'Espagne. Annls Parasit. hum. comp., 50 (1) : 105-122. 
Beaucournu-Saguez F. 1975b. - Récoltes de Simulies (Diptera, Simuliidae) dans le Sud-Est de l'Espagne. Annls Soc, ent. Fr. (N.S.), 11 (1) : 73-89.

Berthélemy C. 1966. - Recherches écologiques et biogéographiques sur les Plécoptères et Coléoptères d'eau courante (Hydraena et Elminthidae) des Pyrénées. Annls. Limnol., 2 (2) : $227-458$

Bouzidi A. 1989. - Recherches hydrobiologiques sur les cours d'eau des massifs du Haut-Atlas (Maroc). Bio-écologie des Macroinvertébrés et distribution spatiale des peuplements. Thèse d'Etat, Université d'Aix-Marseille III. Faculté des Sciences et Techniques de St. Jérôme, $190 \mathrm{p}$.

Clergue-Gazeau M. 1987. - Influence du climat méditerranéen sur la faune simuliidienne (Diptera, Nematocera) d'une rivière pyrénéenne : la Massane. Ecologia Mediterranea, 13 (1-2) : 3-13.

Clergue-Gazeau M. 1991. - Clés de détermination des Simulies (Diptera, Simuliidae) des Pyrénées. Annls. Limnol., 27 (3) : 267-286.

Clergue-Gazeau M. \& Gazagnes G. 1986. - Les Simuliidae de la Neste d'Aure (Pyrénées centrales). I. - Impact des aménagements hydro-électriques dans la haute vallée. (Diptera, Nematocera). Annls Limnol., 22 (2) : 195-203.

Clergue-Gazeau M., Lek So. \& Lek Si. 1991. - Les Simulies d'Afrique du Nord. Nouvelles données sur la répartition de la faune du Maroc et biogéographie des espèces maghrébines (Diptera, Simuliidae). Revue Hydrobiol, trop., 24 (1) : 47-59.

Clergue-Gazeau M. \& Vinçon G. 1990. - Importance de la découverte de Simulium (Rubzovia) lamachi Doby \& David, en Péninsule Ibérique (Diptera : Simuliidae). Nouv. Rev. Entomol. (N.S.), 7 (3) : 303-306.

Couvert J. 1967. - Studi sui Simulidi : Tetisimulium graium n. sp. Boll. Soc. Ent. ital., 97 : 39-49.

Crosskey R.W. 1988. - An annotated checklist of the world blackflies (Diptera : Simuliidae). in : Blackflies, ecology, population management, and annotated world list. Kim (K.C.) \& Merritt (R.W.) ed. Pennsylvania state university, U.S.A.

Dorier A. 1962-1963. - Document pour servir à la connaissance des Simuliidae du Sud-Est de la France. Trav. Lab. Hydrobiol. Grenoble, 7 (2) : 1.15-139.

Giudicelli J. \& Dakki M. 1984. - Les sources du moyen Atlas et du Rif (Maroc) : Faunistique (description de deux espèces nouvelles de Trichoptères), écologie, intérêt biogéographique. Bijdr. Dierk, 54 (1) : 83-100.
Giudicelli J. \& Thierry A. 1985. - About a peculiar type of rheocrene spring in the high Atlas (Marocco). Description of a simuliid characteristic of this habitat, Simulium (Crenosimulium n. sg.) knidirii n. sp. (Diptera, Simuliidae). Bull. Zool. Mus. Univ. Amsterdam., 10 (15) : 109-123.

González-Peña G. 1983. - Factores que determinan la distribución de los Simulidos y Quironomidos (Diptera) de la red hidrografica andorrana. Actas del Primer Congreso Espagnol de Limnologia., 1 : 197-205.

González-Peña G. 1990. - Sistemática y ecología de los Simuliidae (Diptera) de los rios de Catalunya y de otras cuencas hidrograficas españolas. Thèse de Doctorat. Univ. Barcelona. $451 \mathrm{p}$.

González-Peña G., Ferreras Romero M. \& Garcia Rojas A. 1986. - Introducción al estudio de los simulidos (Diptera) de Sierra Morena (Sur de España) : Río Yeguas. Acta de las VII Jornadas $A$ et $E$, Sevilla., 733-744.

Grenier P. \& Bertrand H. 1954. - Simuliidae (Diptera, Nematocera) d'Espagne. Annls Parasti. hum. comp., 29 (4) : 447-459.

Knoz J. 1965. - To identification of Czechoslovakian blackflies (Diptera Simuliidae). Folia Fac. Scient. natur. Univers. Purkyne (Biol. 2), Brno, 6 (5) : 1-54 + 425 planches.

Lavandier P. 1976. - Premières données sur la répartition des Simuliidae (Diptères, Nematocères) dans la vallée d'Aure (Hautes-Pyrénées, France). Bull. Soc. Hist. Nat. Toulouse, $112(1-2)$ : 89-95.

Sanchez-Ortega Y. \& Alba-Tercedor J. 1990. - Los Plecopteros de Sierra Nevada (Granada, España). Zoologica baetica, $1: 77-146$.

Santos-Grácio A.J. 1981. - Estudo dos Simuliideos de Portugal. Bolm. Soc. Port. Ent., 7 (A) : 1-7.

Vinçon G. 1987a. - Comparaison de la faune benthique des vallées d'Aure et d'Ossau, en vue de l'élaboration d'une méthodologie de surveillance des cours d'eau de montagne. Thèse de Docteur-Ingénieur, Toulouse, $n^{\circ} 960,381 \mathrm{p}$.

Vinçon G. 1987b. - Etude hydrobiologique de la vallée d'Ossau (Pyrénées-Atlantiques) II. Le milieu et la structure du peuplement. Annls Limnol., 23 (2) : 95-113.

Vinçon G. \& Clergue-Gazeau . 1988. - Etude hydrobiologique de la vallée d'Ossau (Pyrénées-Atlantiques, France). III. Simuliidae (Diptera, Nematocera) : leur originalité biogéographique et écologique. Annls Limnol., 24 (1) : 67-81. 